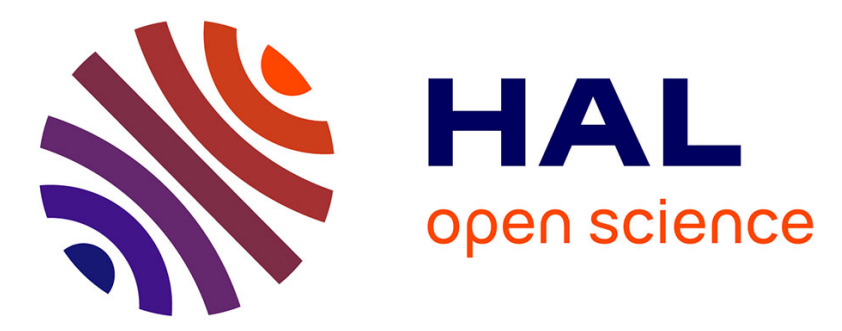

\title{
Autoritarisme, politique symbolique et création musicale. Stratégies de légitimation symbolique dans le Mexique postrévolutionnaire et le Nigeria postcolonial
}

\author{
Luis Velasco-Pufleau
}

\section{- To cite this version:}

Luis Velasco-Pufleau. Autoritarisme, politique symbolique et création musicale. Stratégies de légitimation symbolique dans le Mexique postrévolutionnaire et le Nigeria postcolonial. Revue internationale de politique comparée, 2012, 19 (4), pp.15-40. 10.3917/ripc.194.0015 . hal-01758582

HAL Id: hal-01758582

https://hal.science/hal-01758582

Submitted on 4 Apr 2018

HAL is a multi-disciplinary open access archive for the deposit and dissemination of scientific research documents, whether they are published or not. The documents may come from teaching and research institutions in France or abroad, or from public or private research centers.
L'archive ouverte pluridisciplinaire HAL, est destinée au dépôt et à la diffusion de documents scientifiques de niveau recherche, publiés ou non, émanant des établissements d'enseignement et de recherche français ou étrangers, des laboratoires publics ou privés. 


\title{
AUTORITARISME, POLITIQUE SYMBOLIQUE et CRÉation MUSicale. STratégies de LÉGITIMATION SYMBOLIQUE DANS LE MEXIQUE POSTRÉvolutionNAIRE ET LE Nigeria POSTCOLONIAL
}

\author{
Luis VELASCO-PUFLEAU
}

La politique symbolique, l'instrumentalisation du pouvoir politique à des fins de légitimation par le recours aux procédés symboliques, constitue d'après Max Weber l'une des formes caractéristiques sur lesquelles se fonde la domination légitime ${ }^{1,2}$. Elle a pour objectif de créer le consentement nécessaire à l'institution et à la sauvegarde du pouvoir grâce à l'établissement et au renforcement des croyances et des représentations communes par le biais d'outils symboliques. D'une façon générale, nous envisageons la politique symbolique comme un dispositif ${ }^{3}$ : un ensemble de stratégies hétérogènes, impliquant la production d'objets symboliques et leur mobilisation dans des relations de pouvoir, mises en œuvre par des acteurs et des institutions (État, Église, partis politiques, mouvements sociaux, organisations non gouvernementales) qui visent la légitimation ou la contestation d'un ordre social. Cette définition élargit celle développée précédemment par Pascal Ory ${ }^{4}$, qui

1. Les recherches menant aux présents résultats ont bénéficié d'un soutien financier de l'EHESS pour le projet de recherche «Création musicale, censure et politique symbolique dans les régimes autoritaires et totalitaires ».

2. La typologie proposée par Max Weber est constituée par la domination légale, la domination traditionnelle et la domination charismatique. Néanmoins, la domination légitime repose généralement sur la combinaison de plusieurs de ces trois types à la fois. Voir WEBER M., Économie et société, t.1, Paris, Pocket, 1955, p. 285-336.

3. Michel Foucault définit le dispositif comme « un ensemble résolument hétérogène, comportant des discours, des institutions, des aménagements architecturaux, des décisions réglementaires, des lois, des mesures administratives, des énoncés scientifiques, des propositions philosophiques, morales, philanthropiques, bref : du dit, aussi bien que du non-dit, voilà les éléments du dispositif. Le dispositif luimême, c'est le réseau qu'on peut établir entre ces éléments », $c f$. FOUCAULT M., « Le jeu de Michel Foucault », in Dits et écrits II : 1976-1988, Paris, Gallimard, 2001 [1977], p. 298-329. Cette notion est reprise et analysée par AGAMBEN G. dans Qu'est-ce qu'un dispositif ?, Paris, Payot, 2007 [2006].

4. Pascal Ory distingue la politique symbolique au sein de trois instrumentalisations du pouvoir politique : «l'exercice, intrinsèquement coercitif, de la puissance publique, l'usage, intrinsèquement 
la limite à l'instrumentalisation exclusivement charismatique du pouvoir, au profit d'une conception stratégique de la domination qui regroupe des discours, des pratiques, des objets et des rituels. La politique symbolique, en tant que dispositif, implique ainsi l'ajustement constant de positions et de discours en fonction des exigences et des interactions entre les différents acteurs du champ politique, artistique ou intellectuel. Les enjeux de pouvoir implicites aux représentations et aux actions véhiculées par les stratégies de légitimation peuvent générer des conflits entre les différents acteurs engagés, en fonction des intérêts et des opportunités inhérents au champ social. Les objets symboliques, notamment artistiques, sont engagés au sein de stratégies qui ont recours aux rituels et à l'imaginaire pour transformer la réalité afin de faire reconnaître le pouvoir comme étant légitime. En effet, comme le remarque Georges Balandier, « le pouvoir ne peut s'exercer sur les personnes et sur les choses que s'il recourt, autant qu'à la contrainte légitimée, à des outils symboliques et à l'imaginaire. [...] L'acquiescement, ou le consensus, doivent être provoqués ; il n'y aurait pas d'effets de pouvoir sans les moyens permettant d'obtenir un large (sinon total) consentement. La force légitimée n'y suffit pas, le recours aux dramatisations sociales, à l'imaginaire, à l'efficacité symbolique la complète nécessairement $»^{5}$.

Dans le cas des régimes autoritaires ${ }^{6}$, la mobilisation des ressources symboliques à des fins de légitimation politique est un processus intensifié notamment lors de périodes où les institutions politiques et le sentiment d'appartenance à la communauté nationale sont fragilisés - par exemple après des guerres civiles ou d'indépendance. Le déploiement massif de catégories identificatoires comme celle d' « authenticité », de «peuple» ou de « communauté nationale » par des stratégies de légitimation symbolique obéit ainsi au besoin urgent d'empêcher un nouveau déchaînement ou un retour des violences politiques. En outre, ces stratégies de légitimation symbolique peuvent avoir une dimension nationale ou internationale car les régimes évoluent au sein d'un contexte géopolitique précis et mobilisent des stratégies d'appartenance qui impliquent la désignation des alliés ou des adversaires à l'intérieur et à l'extérieur des frontières. Comme nous le verrons, l'affirmation de la continuité du pouvoir entre un passé idéalisé et

persuasif, de l'action rhétorique, enfin le recours, intrinsèquement charismatique, aux procédés symboliques. À cette dernière seule nous réserverons le terme de politique symbolique », $c f$. ORY P., « L'histoire des politiques symboliques modernes », Revue d'histoire moderne et contemporaine, Paris, $\mathrm{n}^{\circ}$ 47-3, 2000 , p. 525.

5. BALANDIER G., Le Détour. Pouvoir et modernité, Paris, Fayard, 1985, p. 88-94.

6. Nous définissons les régimes autoritaires à partir de la typologie élaborée par Juan Linz : « des systèmes politiques au pluralisme limité, politiquement non responsables, sans idéologie élaborée et directrice mais pourvus de mentalités spécifiques, sans mobilisation politique extensive - excepté à certaines étapes de leur développement - et dans lesquels un leader ou, occasionnellement, un petit groupe exerce le pouvoir à l'intérieur de limites formellement mal définies mais, en fait, plutôt prévisibles » (LINZ J., Régimes totalitaires et autoritaires, Paris, Armand Colin, 2006 [2000], p. 157). 
le présent par le biais de la construction d'une tradition fantasmée peut se révéler d'une efficacité redoutable pour la légitimation politique de régimes autoritaires postcoloniaux, ce qui explique l'utilisation récurrente par ceuxci des stratégies de réactivation symbolique du passé.

Parmi les outils symboliques privilégiés dans les stratégies de légitimation mises en œuvre par les régimes autoritaires se trouve la création musicale, de par sa polysémie et sa capacité à fédérer des émotions au sein de rituels politiques. Pour être efficace, cette utilisation requiert la définition préalable des catégories esthétiques ou politiques construites par un processus de symbolisation, afin de les mobiliser dans des stratégies spécifiques à chaque régime selon un contexte historique donné. Bien que, d'une manière générale, «l'accession au pouvoir politique [soit] à la fois accès à la force des institutions et à la force des symboles ${ }^{7}$, il est indispensable d'interroger en quoi la mobilisation des ressources symboliques par les régimes autoritaires éclaire le mode de fonctionnement de ses institutions dans son rapport au maintien de l'ordre social. En effet, comme le remarque Daniel Bourmaud, "les différents types d'autoritarisme tiennent largement aux stratégies et aux projets de ceux qui exercent le pouvoir. Ces stratégies et ces projets s'inscrivent dans un système construit de représentations, constitutives de l'idéologie $»^{8}$.

Nous proposons dans cet article une analyse ${ }^{9}$ de l'utilisation de la création musicale au sein des stratégies de légitimation symbolique mises en œuvre par des régimes autoritaires postcoloniaux dans des périodes faisant suite à des guerres civiles. Cette analyse comparative se base sur deux études de cas, mettant en perspective les stratégies de légitimation de deux états postcoloniaux multiethniques, gouvernés par des régimes autoritaires fédéraux et centralisés : le régime mexicain postrévolutionnaire durant les années 1930 et le régime militaire nigérian dans les années 1970. Les enjeux de pouvoir des différents acteurs sociaux engagés au sein des dites stratégies occuperont une place centrale dans notre analyse, montrant de quelle manière la musique peut être investie et utilisée pour légitimer ou pour contester l'ordre social. Notre principale hypothèse suppose que l'efficacité à long terme des stratégies de légitimation symbolique mises en place par ces régimes autoritaires est subordonnée à leur intégration dans un dispositif plus large englobant de politiques éducatives, économiques et sociales. La comparaison entre ces deux pays se révèle ainsi extrêmement riche pour comprendre de

7. BALANDIER G., op. cit., 1985 , p. 88.

8. BOURMAUD D., « Aux sources de l'autoritarisme en Afrique : des idéologies et des hommes », Revue Internationale de Politique Comparée, Louvain-la-Neuve, vol. 13, nº 4, 2006, p. 640-641.

9. Basée sur une approche interactive inspirée de celle de la politique du conflit de Charles Tilly: TILLY C. et TARROW S., Politique(s) du conflit. De la grève à la révolution, Paris, Presses de Sciences Po, 2008 [2006]. 
quelle façon les processus de construction nationale conditionnent les stratégies de légitimation symbolique des régimes ainsi que les rôles assumés par les compositeurs et les institutions éducatives et artistiques.

\section{Légitimité, ordre social et politique symbolique}

Notre analyse part du postulat qu'il existe une interdépendance entre les discours développés sur la création musicale et les stratégies de légitimation politique mises en place par les régimes. Les discours produits sur la musique par les différents acteurs (État, compositeurs, critiques, public) et véhiculés par les institutions et les médias, mobilisent des catégories socialement construites qui peuvent être esthétiques, politiques ou morales. Effectivement, dans toute politique symbolique les œuvres et les discours sont engagés au sein de stratégies de légitimation politique qui mettent en scène le pouvoir, investissant ainsi le passé ou le présent par des émotions et des actions jugées légitimes. De même que Denys Cuche, nous pensons que "l'autorité légitime a le pouvoir symbolique de faire reconnaître comme fondées ses catégories de représentation de la réalité sociale et ses propres principes de division du monde social ${ }^{10}$. Nous considérons ainsi que les stratégies de légitimation varient en fonction du contexte sociopolitique et des caractéristiques des régimes, notamment le développement institutionnel d'une idéologie directrice et la capacité de l'État à agir sur la population de son territoire, sur sa répartition, son activité et ses ressources. Parmi les stratégies de légitimation où la création musicale est mobilisée, nous nous intéressons notamment aux rituels politiques ou culturels tels que des commémorations ou des festivals - qui peuvent être diffusés par des médias de masse tels la radio ou la télévision. Ces rituels peuvent être accompagnés ou renforcés par la promulgation de lois pour promouvoir ou censurer certaines pratiques ou certains langages musicaux. De même, ils peuvent s'inscrire dans des projets politiques plus amples, dans la durée et dans l'espace, qui impliquent la création ou la dissolution d'institutions telles que des orchestres, des conservatoires, des unions de compositeurs ou des organes de censure. Comme nous l'analyserons, les modes d'utilisation de la création musicale au sein de ces stratégies dépendent du degré d'investissement préalable de celle-ci par des marqueurs identitaires ou de la volonté de réinvestir les œuvres par des catégories nouvelles.

Les stratégies de légitimation mises en place par le régime mexicain postrévolutionnaire et le régime militaire nigérian trouvent leur origine, de façon explicite ou implicite, dans des événements historiques fondateurs

10. CUCHE D., La notion de culture dans les sciences sociales, Paris, La Découverte, 1996, p. 105. 
qui impliquent une rupture temporelle et territoriale : la Révolution mexicaine (1910-1920) et la guerre civile nigériane (1967-1970). En effet, le Mexique postrévolutionnaire ${ }^{11}$ et le Nigeria des années 1970 ont en commun le fait d'avoir traversé des guerres civiles qui, en plus d'avoir coûté la vie à des millions des citoyens, ont mis en danger l'intégrité des frontières et les institutions des régimes. Dans le cas du Mexique, la Révolution éclate le 20 novembre 1910 pour tenter de mettre fin aux structures coloniales, aux inégalités sociales, et de récupérer les pouvoirs économique, politique et culturel qui sont entre les mains d'une oligarchie tournée vers l'étranger. En première instance, l'objectif de l'insurrection est de renverser la dictature du Général Porfirio Díaz, qui détient le pouvoir depuis trente ans. Le second objectif est de redistribuer les terres et la richesse aux paysans pour en finir ainsi avec près de quatre siècles de domination politique étrangère et d'acculturation forcée ${ }^{12}$. L'inclusion des classes paysannes et des groupes indigènes dans le projet de construction nationale marque un changement profond dans l'histoire du pays. Cependant, la diversité des idéologies et le manque de cohésion politique des chefs révolutionnaires transforment le soulèvement du peuple contre la dictature et l'oligarchie en une sanglante guerre civile à partir de 1915.

Dans le cas du Nigeria, l'indépendance du pays vis-à-vis de la GrandeBretagne - obtenue le $1^{\text {er }}$ octobre 1960 - est suivie de la proclamation de la Première République en 1963, d'une série de coups d'État en 1966 et d'une guerre civile entre 1967 et 1970. Cette guerre est déclenchée par le Gouvernement Fédéral Militaire (GFM) du Nigeria suite à la sécession de la région Est, qui proclame le 30 mai 1967 la République du Biafra et cesse de payer au gouvernement fédéral les taxes (royalties) des revenus qu'elle produit, notamment celles de considérables ressources pétrolières récemment découvertes. L'importance stratégique de ces ressources, la situation géopolitique du pays et la médiatisation de la guerre conduisent la communauté internationale à prendre position dans le conflit armé. Dans un contexte de décolonisation récente et de guerre froide, les puissances occidentales, les pays africains membres de l'Organisation de l'Unité Africaine (OUA), l'URSS, la Chine et Israël soutiennent diplomatiquement ou militairement l'un de deux camps. Des organisations internationales non gouvernementales - tels l'Église catholique et la Croix-Rouge - acheminent de l'aide alimentaire à la région pour combattre la famine. Les compromis forcés entre

11. La période postrévolutionnaire s'étend de la fin de la Révolution mexicaine en 1920 à l'institutionnalisation de celle-ci dans la consolidation du régime autoritaire mexicain en 1940.

12. Le Mexique obtient son indépendance vis-à-vis de l'Espagne en 1821 et devient ainsi le cinquième État latino-américain, après le Venezuela (1811), l'Argentine (1816), le Chili (1818) et la grande Colombie de Bolivar (1819), à se constituer sur les ruines du vieil empire des Habsbourg. Cependant, les structures socioéconomiques fortement hiérarchisées et les rapports communautaires établis durant la période coloniale restèrent en place. 
la Croix-Rouge et le gouvernement fédéral nigérian mènent son action dans une impasse, provoquant une scission à l'origine d'une nouvelle forme d'action humanitaire développée par différentes organisations dans les décennies suivantes ${ }^{13}$. La guerre s'achève le 14 janvier 1970 avec la défaite et la réincorporation du Biafra au Nigeria par le régime militaire de Yakubu Gowon (1966-1975), avec un bilan humain très lourd : entre un et trois millions de morts et environ trois millions de déplacés ${ }^{14}$. À l'issue de la guerre civile, le gouvernement militaire engage une transition du pouvoir vers un régime civil qui donnera lieu à la Deuxième République (1979-1983).

Dans les deux pays étudiés, le maintien de la paix après la guerre civile se fonde sur la reconnaissance partagée de la légitimité des autorités issues du conflit. Cette reconnaissance nécessite, en plus du contrôle par le régime des forces armées et de la police, de la réactivation de l'économie et de la consolidation des institutions politiques, la mobilisation de ressources symboliques capables de fédérer une communauté imaginaire ${ }^{15}$. Cependant, les deux pays présentent des configurations politiques extrêmement différentes, en particulier à propos de la capacité de l'État central à agir sur la population des États fédérés afin d'enclencher un processus de construction nationale et d'inculquer un sentiment d'appartenance commune par le biais de l'éducation. En effet, comme le remarque Anne-Marie Thiesse, l'éducation constitue le moyen le plus efficace pour transmettre la langue, l'histoire et la géographie de la Nation ainsi que pour enseigner comment «être et penser nationalement ${ }^{16}$.

Le Mexique s'appuie sur un solide fédéralisme ${ }^{17}$ pour mener une vaste campagne d'éducation populaire et esthétique, conduite par la Secretaría de Educación Pública ${ }^{18}$ (Ministère de l'Éducation Publique, SEP), afin

13. Suite à cette scission est fondé en 1969, sous l'égide de Bernard Kouchner, le Comité de lutte contre le génocide du Biafra, qui devient en 1971 l'ONG Médecins sans frontières (MSF). Sur le rôle et les enjeux de la France dans la médiatisation de la guerre civile nigériane voir LAVOINE Y., « Médecins en guerre : du témoignage au "tapage médiatique" (1968-1970) », Le Temps des médias, n 4, 2005, p. 114-126.

14. FALOLA T., HEARTON M., A History of Nigeria, New York, Cambridge University Press, 2008, p. 180. Toutes les citations originales en anglais et en espagnol ont été traduites par les soins de l'auteur. 15. Dans le sens donné par Benedict Anderson au concept de «nation », « une communauté politique imaginaire, et imaginée comme intrinsèquement limitée et souveraine. Elle est imaginaire (imagined) parce que même les membres de la plus petite des nations ne connaîtront jamais la plupart de leurs concitoyens : jamais ils ne les croiseront ni n'entendront parler d'eux, bien que dans l'esprit de chacun vive l'image de leur communion », ANDERSON B., L'imaginaire national. Réflexions sur l'origine et l'essor du nationalisme, Paris, La Découverte, 1996 [1983], p. 19.

16. THIESSE A.-M., La création des identités nationales, Paris, Le Seuil, 1999, p. 240.

17. Le Mexique est une république fédérale par intermittence depuis 1824 . Toutefois, le fédéralisme est consolidé et garanti par la constitution de 1917, rédigée durant la dernière période de guerre civile par le gouvernement du Général Venustiano Carranza (1859-1920) - premier gouvernement issu de la Révolution.

18. Fondé en septembre 1921 par le philosophe José Vasconcelos (1882-1959), le Ministère de l'Éducation Publique est structuré en trois départements : le Département Scolaire (chargé des programmes 
d'homogénéiser et d'unifier la communauté nationale avec une langue et un passé communs tout en excluant les différences culturelles. L'État mexicain postrévolutionnaire impose un nouveau discours identitaire qui place le « métissage » en tant que modèle identificatoire unificateur et considère l'assimilation des populations indigènes au sein de celui-ci comme la condition de la consolidation nationale. Tous les efforts dans le domaine de l'éducation des gouvernements postrévolutionnaires portent ainsi sur "l'intégration » des minorités ethniques dans une supposée communauté nationale. Dans un article paru en 1933 dans la revue du Ministère de l'Éducation Publique, El Maestro Rural, la fédéralisation de l'éducation et l'assimilation de tous les peuples dans l'unité nationale sont considérées comme « la condition de base, indispensable, de nature à résoudre tout le problème national : l'intégration des différents groupes ethniques dans une ample et cohérente unité, à l'intérieur de laquelle se concilient les différences et les oppositions raciales ; l'annulation totale de l'isolement des petites communautés ; l'extraction du traditionnel régionalisme de tous les lieux et son incorporation à l'esprit profond de la nationalité ${ }^{19}$.

La SEP élabore à Mexico, la capitale fédérale, les programmes d'éducation qui sont mis en œuvre dans les 31 États de la République, permettant au régime de contrôler l'écriture et la transmission de l'Histoire de la Nation. Le dispositif de politique symbolique mis en place par le régime postrévolutionnaire mexicain au sein du processus de construction nationale implique également l'élaboration d'un art nationaliste qui serait capable de rivaliser avec l'art européen. Les principales catégories esthétiques investies par le régime concernant la musique font référence à l'authenticité et au contenu nationaliste des œuvres - fondés sur les notions de « race » et de «métissage » promues par une idéologie « indigéniste »-en fonction de leur matériau et de leur capacité supposée à construire la nouvelle Nation issue de la Révolution. Les principaux acteurs de cette catégorisation sont les compositeurs et, dans une moindre mesure, les critiques musicaux. Ces acteurs sont engagés au sein du champ artistique dans une lutte pour le contrôle des institutions musicales, notamment le Conservatoire National, l'Orchestre Symphonique du Mexique (OSM) et le Département des Beaux-Arts de la SEP. Parmi les compositeurs, Carlos Chávez ${ }^{20}$ joue un rôle central dans

d'éducation), le Département des Bibliothèques (chargé de l'édition des livres et manuels d'étude) et le Département des Beaux-Arts (chargé de coordonner la production et la diffusion culturelle). Le programme d'éducation populaire développé par Vasconcelos à la tête de la SEP s'inspire de la façon de procéder sur le terrain des missions coloniales catholiques et du modèle soviétique créé par Anatoli V. Lounatcharski, commissaire du peuple à l'Instruction publique sous Lénine, cela malgré les réticences manifestées par le ministre mexicain envers l'idéologie marxiste.

19. Cité dans FLORESCANO E., Historia de las historias de la nación mexicana, México, Taurus, 2004, p. 415.

20. Le compositeur mexicain Carlos Chávez (1899-1978) devient le directeur de l'Orchestre Symphonique du Mexique (OSM) et du Conservatoire National en 1928, occupant dorénavant une position 
l'institutionnalisation de ces catégories. Il applaudit dès 1925 l'initiative du ministre de l'Éducation consistant à créer un département au sein de la SEP - la Dirección de Cultura Estética - destiné à « susciter des traits de style national au sein du [public] non spécialisé, s'adressant principalement aux classes ouvrières de la ville $»^{21}$. Une fois directeur du Conservatoire National, il affirme que «l'éducation musicale doit avoir comme principale finalité de faire partie de l'éducation intégrale de l'individu $»^{22}$. Néanmoins, concernant le contenu de cette éducation populaire par le biais de l'art, Chávez conteste que « le travail socialisateur de l'art consiste à adopter le type d'art des masses non cultivées ou incultes. Au contraire, le travail socialisateur doit consister à imposer la beauté de l'art supérieur aux masses qui l'ignorent et le comprennent mal ${ }^{23}$. Ainsi, les catégories d'évaluation de Chávez perpétuent la violence symbolique exercée au sein de la société postcoloniale mexicaine. Il déplace les conceptions coloniales - qui ont justifié la domination esthétique par une hypothétique supériorité de l'art européen sur l'art préhispanique - vers celles de la supériorité de l'art savant sur l'art populaire, tout en légitimant l'utilisation de ce dernier dans la création nationaliste dirigée par l'État. En effet, il considère que la musique « est une activité d'intérêt national » et qu'elle doit être le «lien puissant qui unifie l'âme des Mexicains $»^{24}$. L'art et la musique seraient ainsi « un phénomène humain que l'État doit développer et utiliser comme arme éducative en direction des classes prolétaires ${ }^{25}$.

En revanche, le Nigeria ne vise pas une construction nationale par le biais d'une assimilation des peuples préexistants dans une communauté nationale homogène. Le pays est constitué au moment de son indépendance par trois

dominante au sein du champ artistique mexicain grâce à sa capacité à s'adapter aux exigences idéologiques et politiques du régime. Il sera le fondateur et le premier directeur de l'Institut National des Beaux-Arts (INBA) en 1947. Sur l'adaptation du discours idéologique de Chávez aux exigences du régime autoritaire postrévolutionnaire voir VELASCO PUFLEAU L., « Nacionalismo, autoritarismo y construcción cultural: Carlos Chávez y la creación musical en México (1921-1952) », in ILLIANO R., SALA M. (eds), Music and Dictatorship in Europe and Latin America, Turnhout, Brepols, 2009, p. 707730.

21. CHÁVEZ C., « México y la música », El Globo, México, 25 février 1925, publié dans CHÁVEZ C., Escritos periodísticos (1916-1939), México, El Colegio de México, 1997, p. 81-85.

22. CHÁVEZ C., «La música, la universidad y el Estado », El Universal, México, 3 juillet 1929, publié dans CHÁVEZ C., op.cit., 1997, p. 121-127.

23. CHÁVEZ C., « Una nueva actividad del Conservatorio Nacional », El Universal, México, 6 septembre 1929, publié dans CHÁVEZ C., op. cit., 1997, p. 141-143.

24. CHÁVEZ C., «El Conservatorio en 1929 », El Universal, México, 2 janvier 1930, publié dans CHÁVEZ C., op. cit., 1997, p. 147-148.

25. CHÁVEZ C, «Arte proletario », El Universal, 29 septembre 1934, publié dans CHÁVEZ, op. cit., 1997, p. 259. Presque vingt ans plus tard, Chávez continue à considérer l'État comme «l'institution nationaliste par excellence » affirmant qu' « en matière d'art, [il] doit poursuivre : le développement d'un art propre, la connaissance de l'art universel et la protection de l'art national », CHÁVEZ C., « Dos años y medio del INBA », in GARCÍA MORILLO R., Carlos Chávez. Vida y obra, México, FCE, 1960, p. 148. 
grandes régions héritées de la division coloniale britannique et fondées sur des bases majoritairement ethniques : Haoussa dans le Nord, Yoruba dans l'Ouest et Ibo dans l'Est. En effet, « la nature et la particularité de l'administration coloniale britannique encourage le développement au Nigeria d'une théorie du fédéralisme basée sur une théorie des nationalités ou des groupes ethniques ${ }^{26}$. Ces régions sont fragmentées par décret du Gouvernement Militaire Fédéral de Yakubu Gowon en douze États le 27 mai 1967 mais ne deviennent une réalité politique et administrative qu'après la fin de la guerre civile en 1970. Les bénéfices de cette division sont considérables pour le gouvernement central et pour les nouveaux gouvernants des États fédérés. D'une part, les revenus pétroliers sont distribués par l'État central aux États fédérés, bénéficiant aux nouvelles élites dirigeantes et créant des nouvelles bureaucraties. D'autre part, le gouvernement central bénéficie d'un renforcement de sa domination grâce à l'accroissement de la présence de l'armée dans le pays et aux nouvelles allégeances des autorités traditionnelles locales. Après le coup d'État qui renverse Gowon en 1975, les régimes de Murtala Muhammed (1975-1976) et d'Olusegun Obasanjo (1976-1979), s'engagent à respecter le fédéralisme comme garant du caractère pluriel de la société afin d'éviter le déchaînement de nouvelles violences. La création de sept nouveaux États par Muhammed en février 1976 confirme cette volonté, en répondant partiellement aux demandes toujours plus nombreuses des autorités locales qui voient dans la création d'un État le moyen d'accéder aux rentes pétrolières, de dynamiser l'économie et d'accroître leur influence politique ${ }^{27}$. De fait, l'instrumentalisation de la création de nouveaux États à des fins de légitimation du pouvoir des militaires est la grande caractéristique des années 1970-1999.

Concernant l'éducation nationale, ce n'est qu'en septembre 1976 que le régime militaire nigérian lance le programme d'Éducation primaire universelle (Universal Primary Education program, UPE), rendant l'éducation gratuite aux enfants de six ans. La création de l'UPE constitue la première réforme d'envergure du système d'éducation héritée de la période coloniale, les efforts précédents pour alphabétiser la population ayant échoué en raison du manque des moyens humains et financiers des régions ${ }^{28}$. Ainsi, l'absence d'un système d'éducation solide à l'échelle du pays ne favorise

26. JINADU L.-A., « The Constitutional Situation of the Nigerian States », Publius, vol. 12, $\mathrm{n}^{\circ}$ 1, 1982, p. 158.

27. Daniel Bach souligne que « la création d'un nouvel État signifie l'établissement d'une fonction publique locale, la distribution de contrats pour la construction d'un nouveau secrétariat, de nouvelles routes et infrastructures, d'hôpitaux, d'écoles, de logements, voire d'une université, sans parler de la mise en place d'entreprises étatiques, d'une station de télévision et d'un quotidien ». BACH, D., « Les effets pervers du fédéralisme nigérian », Politique africaine, n 32, 1988, p. 25-26.

28. Sur la place de l'ethnicité dans le système d'éducation nigérian avant et après l'indépendance voir AGUOLU C., « The Role of Ethnicity in Nigerian Education », The Journal of Negro Education, vol. $48, \mathrm{n}^{\circ} 4,1979$, p. $513-529$. 
pas la construction d'une communauté nationale, d'autant plus que les allégeances aux autorités locales sont proportionnelles à la méfiance que suscite le gouvernement central. De fait, la nécessité de promouvoir l'appartenance nationale est soulignée par le Constitutional Drafting Committee ${ }^{29}$ (CDC) dès 1976, lequel déclare que la condition pour préparer le retour à un gouvernement civil est la «promotion de la loyauté nationale dans une société multiethnique ». Tandis que par souci de réconciliation nationale après la guerre civile le Gouvernement Militaire Fédéral refuse de célébrer la réunification du pays, le CDC affirme que « l'État doit favoriser un sentiment d'appartenance et de participation entre les différentes parties du pays, afin que la fidélité à la Nation prévale sur les loyautés locales ${ }^{30}$. Le gouvernement fédéral impose ainsi en 1976 à chaque écolier de réciter au début et à la fin de la journée un serment d'allégeance à la Nation : « Je m'engage envers mon pays le Nigeria, à être fidèle, loyal et honnête, à servir le Nigeria de toutes mes forces, à défendre son unité et respecter son honneur et sa gloire ; aide-moi Seigneur ${ }^{31}$. Néanmoins, étant donné que la préservation et la pérennisation du régime nigérian dépendent de la caution politique des autorités traditionnelles des différentes régions, l'homogénéisation de la population du territoire par l'imposition d'une culture hégémonique représentative de la communauté nationale s'avère impossible ${ }^{32}$. De ce fait, le régime nigérian promeut la diversité et l'hétérogénéité des manifestations identitaires en évitant de déprécier la pluralité des traditions artistiques du pays devant une supposée supériorité des traditions européennes. Le régime organise ainsi avec succès à partir de 1970 un Festival National des Arts et de la Culture (National Festival of Arts and Culture, NAFEST) afin de présenter les manifestations artistiques et culturelles des États fédérés sélectionnées préalablement dans des festivals locaux. Conçu par le Nigerian Arts Council - ancêtre du National Council for Arts and Culture (NCAC) - comme une stratégie pour promouvoir l'unité nationale après la guerre civile, le festival devient un « véritable étendard de la célébration de l'unité et de la diversité du Nigeria ${ }^{33}$. De même, le gouvernement fédéral vise la consolidation de l'unité nationale avec la publication en 1977 du National Policy on Education (NPE), qui encourage l'apprentissage des langues

29. Crée par Murtala Muhammed en octobre 1975, le Constitutional Drafting Committee (CDC) est un groupe restreint d'experts chargés de préparer un projet de constitution pour le soumettre à l'Assemblée Constituante, un organe élu chargé de proposer des modifications et de ratifier la nouvelle constitution. 30. JOSEPH R., Democracy and prebendal politics in Nigeria: The rise and fall of the second republic, Cambridge, Cambridge University Press, 1987, p. 43, cité dans APTER A., The Pan-African Nation. Oil and the Spectacle of Culture in Nigeria, Chicago, University Chicago Press, 2005, p. 45.

31. Cité dans BRAY T. et COOPER G., «Education and Nation Building in Nigeria since the Civil War », Comparative Education, vol. 15, n 1, 1979, p. 36.

32. Pour une analyse du rôle des autorités traditionnelles dans la vie politique du Nigeria voir VAUGHN O., « Les chefs traditionnels face au pouvoir politique », Politique africaine, Bordeaux, n 32, 1988, p. 44-56.

33. http://www.ncac.gov.ng/nafest.html, page consultée le 26 mai 2012. 
majeures du pays (haoussa, ibo et yoruba) - en plus de l'anglais, langue officielle de l'administration fédérale - de l'enseignement primaire jusqu'à l'université.

Ces projets de construction nationale façonnent les dispositifs, les modalités, les visées et les catégories identitaires mobilisés par les stratégies de légitimation symbolique des régimes mexicain et nigérian, notamment les rituels politiques destinés à réactiver le passé par la sacralisation d'une mémoire historique nationale. De ce point de vue, la musique utilisée dans ces rituels mémoriels symbolise d'une certaine façon la communauté esthétique que les régimes souhaitent fédérer et inciter à l'action par le biais d'un processus d'identification au pouvoir lui-même.

\section{Rituels politiques et création musicale : réactivation du passé et légitimation du présent}

Les rituels politiques occupent une place particulière au sein des stratégies de légitimation des régimes par leur capacité à transformer symboliquement la réalité. Comme l'affirme Claude Rivière, « chaque régime politique se dote d'un système de représentations idéologiques qu'il tient pour scientifique. Il formule ses impératifs et crée ses rites séculiers à fonction mobilisatrice ou thérapeutique ${ }^{34}$. L'efficacité symbolique des rituels dépend des croyances et des catégories mobilisées dans l'espace public. Dans le cas du Mexique postrévolutionnaire, le régime puise sa légitimité dans la Révolution mexicaine : elle est l'événement fondateur de la nouvelle Nation et tous les efforts du régime se concentrent pour réussir son identification à cette Nation. Ainsi, la musique qui se fonderait sur une continuité entre le passé préhispanique, la période coloniale et l'événement révolutionnaire serait capable de représenter la nouvelle Nation. Cette idée est exprimée dès 1930 par le compositeur Carlos Chávez, alors directeur du Conservatoire National et directeur de l'Orchestre Symphonique du Mexique (OSM), qui affirme que « la Révolution Mexicaine a établi les bases d'une nouvelle conscience nationale ; les civilisations pré-cortésienne et européenne, qu'on a considérées antagonistes durant des siècles, donnent maintenant comme résultat magnifique, dans la Révolution actuelle, le fruit de l'expression artistique de notre race ${ }^{35}$.

34. RIVIÈRE C., Les liturgies politiques, Paris, PUF, 1988, p. 145.

35. CHÁVEZ C., « Carta abierta a la juventud », Música. Revista mexicana, México, vol. 1, $\mathrm{n}^{\circ} 1$, avril 1930, p. 3. 
Ainsi, le régime mexicain postrévolutionnaire du Général Lázaro Cárdenas ${ }^{36}$ intensifie à partir de 1934 sa mobilisation de ressources symboliques dans des actes commémoratifs orientés vers la réécriture et l'institutionnalisation de l'Histoire révolutionnaire récente. Ce travail de réécriture est réalisé, comme le montre l'historien Enrique Florescano, par le biais d'une " succession de commémorations, rites, monuments et célébrations qui envahissent les différents espaces de la vie publique ${ }^{37}$, c'est-à-dire par la consolidation d'une politique symbolique de grande ampleur. Parmi les principaux rituels mémoriels organisés par le nouveau régime, afin d'assurer symboliquement la transition politique et la continuité du pouvoir tout en évitant un nouveau conflit armé ${ }^{38}$, trouve place la « Cérémonie en mémoire du Général Obregón ${ }^{39}$ présidée par le Président Cárdenas en personne ${ }^{40}$. Cette cérémonie a lieu le 17 juillet 1935, date du septième anniversaire de l'assassinat de l'ex-président Álvaro Obregón dans le parc de La Bombilla, en plein cœur de Mexico. Elle a pour fonction de consacrer la Révolution tout en la reliant au régime afin d'y puiser sa légitimité - et de créer un sentiment d'unité nationale en effaçant les profondes divisions de la « famille révolutionnaire » durant la guerre civile et la période postrévolutionnaire. L'Orchestre Symphonique du Mexique (OSM) prend part à la cérémonie en interprétant deux œuvres du compositeur Silvestre Revueltas ${ }^{41}$ - Caminos et Janitzio, sous la direction de Carlos Chávez -, alors co-directeur de l'OSM et collaborateur de Chávez jusqu'en 1935. Les œuvres de Revueltas sont perçues par le public comme profondément mexicaines et certains critiques n'hésitent pas à voir dans le compositeur le «musicien représentatif

36. Lázaro Cárdenas (1895-1970), Général et président du Mexique de 1934 à 1940. Il déclare son gouvernement de nature socialiste et exproprie les compagnies étrangères d'un grand nombre de secteurs (pétrole, minéraux, électricité). En 1936, il donne l'autorisation à l'asile politique de Léon Trotsky et, en 1939, à l'accueil des exilés républicains espagnols.

37. FLORESCANO E., op. cit., 2004, p. 398.

38. L'arrivée au pouvoir du Général Lázaro Cárdenas le $1^{\text {er }}$ décembre 1934 marque la fin du Maximato, période de forte instabilité politique comprise entre 1929 et 1934 caractérisée notamment par un pluralisme politique limité et par la persistance d'une répression religieuse et syndicale : le Parti Communiste Mexicain (PCM) et son journal El Machete sont interdits, la persécution religieuse prolongée et les activités syndicales réprimées. Le Maximato (Période gouvernée par le Chef Suprême), tire son nom de la référence à l'influence Plutarco E. Calles (1877-1945), ancien Ministre de l'Intérieur du gouvernement Obregón et ex-président du Mexique (1924-1928) - nommé alors le Jefe Máximo de la Révolución (Chef Suprême de la Révolution) - qui gouverne le pays en sous-main.

39. Ancien ministre de guerre du gouvernement du Général Venustiano Carranza, le Général Álvaro Obregón (1880-1928) combat Francisco «Pancho » Villa (1878-1923) et Emiliano Zapata (1879-1919) durant la dernière étape révolutionnaire. Il est président du Mexique de 1920 à 1924.

40. El Nacional, México, 13 juillet 1935.

41. Silvestre Revueltas (1899-1940) est l'un des compositeurs mexicains les plus importants de la première moitié du XX ${ }^{\mathrm{e}}$ siècle. Co-directeur de l'OSM avec Chávez entre 1929 et 1935, il rompt avec lui et s'engage dans l'antifascisme communiste au sein de la Ligue des Écrivains et Artistes Révolutionnaires (LEAR). Il voyage en Espagne en 1937 durant la guerre civile et dirige sa musique dans des actes antifascistes républicains. Sur ce compositeur voir VELASCO PUFLEAU L., « Musique et antifascisme : Silvestre Revueltas dans le contexte de la Guerre civile espagnole », Textuel, n 63, 2010, p. 77-93. 
du Mexique moderne, du Mexique où l'ancienne tradition et le sentiment moderne se donnent la main ${ }^{42}$. La musique de Revueltas participe à la surcharge de sens de l'énorme monument érigé à la mémoire du Général révolutionnaire, situé sur le lieu de sa mort, mobilisant un dispositif émotionnel à caractère nationaliste. Comme le souligne Philippe Braud, avec les pratiques rituelles et cérémonielles « l'insertion du symbolique dans l'univers physique atteint un degré particulièrement élevé de réalité puisqu'elles se déploient dans un espace qu'elles dynamisent, puisqu'elles mobilisent des lieux, des objets, des individus $\gg{ }^{43}$. Ce monument, pour extraordinaire qu'il puisse paraître, est destiné à accueillir le bras arraché à Obregón par les troupes de Francisco Villa pendant la Révolution lors de la meurtrière bataille de Celaya en $1915^{44}$. L'intervention de l'OSM accomplit alors une fonction rituelle de sacralisation de la relique dans un cadre d'institutionnalisation de la nouvelle mémoire historique révolutionnaire.

Quelques semaines plus tard, l'OSM collabore à nouveau à un acte mémoriel du régime de Cárdenas : l'inauguration de la plaque commémorative du fondateur du journal El Nacional, « l'illustre Réformateur José María Mora » ${ }^{45}$. En effet, l'OSM interprète à nouveau Caminos (sous la direction de Chávez) et Janitzio (sous la direction de Revueltas) lors de la cérémonie qui a lieu le 10 août à l'endroit où se trouvait le siège du journal à Mexico. À cette occasion, la municipalité change le nom de l'ancienne rue de San Diego pour celui du Dr. Mora, considéré par le nouveau régime comme l'un des précurseurs idéologiques de la Révolution. Pour El Nacional, la plaque exalte l'endroit « où s'érige fièrement le bâtiment du porteparole de la Révolution, où incubent les idées et se protègent les droits de la classe prolétarienne ${ }^{46}$. Les orateurs se succèdent sur le podium, racontant comment le Dr. Mora, « le civilisateur, affronte aussi bien les pouvoirs rétrogrades que les masses irresponsables et ses caudillos $^{47}$ démagogues et illettrés, en profitant de la plus petite percée pour filtrer sa lumière et injecter des nouveautés dans l'immense chaos ${ }^{48}$. Les critiques du journal applaudissent l'intervention de l'OSM et les œuvres de Revueltas, attribuant à Caminos une « authentique saveur révolutionnaire ${ }^{49}$. En effet, le

42. KAHAN S., « La màs completa "incompleta” bella obra de Revueltas », El Universal Gráfico, México, 6 juin 1933.

43. BRAUD P., L'émotion en politique, Paris, Presses de Sciences Po, 1996, p. 131.

44. La relique sera exposée au public dans un bocal de formol au centre du monument durant des années avant d'être remplacée par une copie en bronze.

45. El Nacional, México, 10 août 1935. Le libéral mexicain José María Mora (1794-1850) contribue au mouvement de réforme qui aboutit à la séparation entre l'Église et l'État, inscrite dans la Constitution mexicaine de 1857.

46. El Nacional, México, 11 août 1935.

47. Leaders politiques charismatiques, généralement issus de l'armée, exerçant un pouvoir autoritaire.

48. El Nacional, México, 11 août 1935.

49. Ibid. 
matériau de Caminos partage avec Janitzio la forte influence des mélodies et rythmes issus de la musique paysanne métisse, sans pourtant être basée sur un thème traditionnel précis. Généralement, Revueltas utilise la citation courte des thèmes métis, les transforme ou en invente certains qui pourraient passer pour d'authentiques thèmes paysans. La plupart de ces thèmes sont issus des genres hérités de l'Espagne du XVII ${ }^{e}$ siècle et transformés au Mexique, notamment le son ${ }^{50}$. Janitzio peut être classée dans une catégorie de musique mexicaine qui puise son identité nationaliste dans la musique métisse connue par la plupart des habitants du pays. L'insertion des thèmes paysans n'obéit pas à un désir d'élever la musique populaire à un certain modèle savant, mais à celui d'amplifier les caractéristiques de cette musique avec les ressources de l'orchestre symphonique et des techniques issues de la tradition occidentale (polyphonie, dissonances et superpositions tonales, travail sur la texture et la couleur orchestrale, etc.). Grâce à sa capacité de synthétiser symboliquement les attributs officiels de la nouvelle nation métisse, Janitzio jouit d'une réception inégalée dans le catalogue du compositeur, et cela dès sa création le 8 décembre 1933 durant la saison de l'OSM. En effet, à peine trois jours après sa création, l'œuvre sera définie par Salomón Kahan, critique musical du journal El Universal Gráfico, comme le « point culminant dans la création de Revueltas », qui n'hésite pas à affirmer que « si pour une quelconque raison sa création stagnait, Janitzio lui assurerait une place d'honneur dans l'histoire de la musique mexicaine $»^{51}$. De même, le chaleureux accueil de la part du public se confirme à la fin de la saison de l'OSM, quand Janitzio arrive à la première place du vote pour choisir le programme du "Concert Extraordinaire de Clôture » de la saison de l'OSM. Ainsi, le 22 décembre 1933 Janitzio est interprétée à nouveau avec les autres œuvres lauréates : Le Pins de Rome de Respighi, L'Oiseau de feu de Stravinsky et le Concerto pour piano en La mineur de Schuman, avec Claudio Arrau comme soliste ${ }^{52}$. Cet accueil, une première pour une œuvre mexicaine contemporaine, est influencé incontestablement par le discours identitaire officiel qui plaçait dès 1930 le métissage comme la condition et l'évolution ultime de la culture nationale.

Ainsi, l'utilisation de la musique dans le cadre de pratiques cérémonielles du régime Cárdenas - en tant que catalyseur identitaire - obéit à une volonté de mobiliser l'ensemble des dispositifs sensoriels afin d'exacerber la dimension émotionnelle du rituel. Les rituels mémoriels mis en place par

50. Genre vocal-instrumental d'origine espagnole proche de la guajira, le fandango, le zapateado et la petenera, il est caractérisé par l'alternance métrique 3/4 et 6/8 dans un tempo vif. Cependant, il prit au Mexique des caractéristiques particulières sous les influences indigènes (expression vocale, technique instrumentale) et africaines (introduction des percussions, polyrythmie).

51. El Universal Gráfico, México, 12 décembre 1933.

52. Cf. le programme du Concierto Extraordinario de Clausura de la saison 1933 de l'OSM, 22 décembre 1933 au Teatro Hidalgo. 
le régime mexicain peuvent être considérés comme des rites d'institution du passé, le réactivant et le consacrant dans le présent par un dispositif symbolique. La musique structure temporellement le rituel et participe à la construction d'un imaginaire national ancré dans le temps et dans l'espace. La participation du public aux rituels le fait communier avec la communauté imaginaire et adhérer aux catégories instaurées par le biais d'un processus d'identification. Les interventions successives de l'OSM accomplissent la fonction de sacralisation de la nouvelle mémoire historique révolutionnaire au sein de ces rituels par la mobilisation de références symboliques précises. Cependant, ceci devient possible grâce à l'encouragement officiel d'un art qui représenterait le Mexique ancien et moderne ainsi que grâce à l'identification de la part des différents acteurs d'une musique perçue comme nationaliste (représentative de la communauté nationale). Dans le cas du Nigeria, le régime expose la diversité des manifestations identitaires comme une mosaïque nationale sans soutenir un art savant national qui les dépasserait par un processus d'inclusion. Néanmoins, les deux régimes cherchent à souligner l'hypothétique continuité de leur pouvoir avec celui des autorités précoloniales afin d'y puiser leur légitimité.

Le régime militaire nigérian organise à Lagos, entre le 15 janvier et le 12 février 1977, un immense festival artistique accompagné d'un colloque international où participent des intellectuels noirs du monde entier : le Second World Black and African Festival of Arts and Culture (FESTAC) ${ }^{53}$. $\mathrm{Ce}$ festival constitue un dispositif de politique symbolique avec une double fonction de légitimation nationale et internationale. Pour le régime, le FESTAC constitue « un grand moment dans l'histoire des peuples noirs et africains dans le monde. [...] Un moment où nos peuples doivent montrer de façon positive qu'ils veulent la liberté et l'autonomie culturelle sans hypocrisie ; qu'ils veulent la justice sociale et les droits politiques sans exploitation, et enfin, qu'ils veulent clairement et sans équivoque l'identité culturelle de leur existence objective dans notre monde $\gg^{54}$.

L'enjeu de légitimation interne est d'une importance considérable pour le régime car le Nigeria connaît depuis la guerre civile (1967-1970) une instabilité politique accrue marquée par une succession de coups d'État. Les préparatifs du FESTAC débutent durant le régime de Yakubu Gowon avec l'inauguration à Lagos en novembre 1973 du siège du Comité International du FESTAC, en présence du président du Sénégal Léopold Sédar Senghor. A son arrivée au pouvoir en 1975, Murtala Muhammed crée une commission

53. Le premier festival mondial des arts nègres ayant eu lieu à Dakar en 1966, sous l'impulsion de Léopold Sédar Senghor et sous la direction de l'intellectuel sénégalais Alioune Diop, fondateur de la revue Présence Africaine.

54. FINGESI O., «Introduction », in Festac'77, Lagos, Africa Journal Ltd. and The International Festival Committee, 1977, p. 9. 
pour enquêter sur la corruption et les dépenses immenses engagées pour les préparatifs du FESTAC, voulant ainsi se démarquer des pratiques de son prédécesseur. Après l'assassinat de Muhammed, Olusegun Obasanjo accède au pouvoir et mène à terme le FESTAC grâce aux revenus pétroliers. La réalisation du festival obéit à une logique de légitimation symbolique du pouvoir qui n'est pas remise en cause par les différents coups d'État, Muhammed et Obasanjo étant conscients de l'importance et de l'efficacité symbolique de l'entreprise commencée par Gowon. L'organisation du FESTAC veut accomplir le dessein de légitimer la Nation dans une optique panafricaine qui s'enracinerait dans un socialisme communautariste originaire du continent africain. Ainsi, la manifestation de la diversité des formes artistiques du pays est mise à l'honneur lors du FESTAC, à la façon d'une exposition coloniale, accompagnée d'une rhétorique d'unité symbolique du destin national. Pour Obasanjo, le festival constitue l'" opportunité de raconter les exploits de nos ancêtres et de nos contemporains ${ }^{55}$. Le régime cherche, par la mise en scène de son pouvoir, à instaurer une hypothétique unité nationale qui émergerait de la fragmentation imposée par la domination coloniale. Dans cette optique, les responsables politiques dénoncent l'héritage colonial en reconstruisant un passé idéalisé et en exprimant la nécessité d'extirper les éléments exogènes qui nuisent à la culture africaine et engendrent de la violence et de la corruption. Le président du Comité International du Festival, le Commandant O. P. Fingesi, déclare que « l'assimilation aveugle d'éléments étrangers dans la vie culturelle autochtone de la société africaine a intensifié la crise dans notre vie sociale actuelle. Par exemple, des institutions comme l'éducation, le mariage, le système de la famille et même nos modes de comportement ont été envahis par des influences étrangères. L'ampleur de la délinquance, à la fois des adultes et des jeunes, et d'autres vices sociaux exprimés dans les différentes formes de comportement social déviant comme la subornation et la corruption sont les effets malencontreux du conflit entre la culture de la tradition africaine et la civilisation occidentale ${ }^{56}$.

Ainsi, le régime militaire nigérian mobilise le mythe d'une intégrité idéalisée des sociétés africaines précoloniales en imputant la corruption et les problèmes sociaux contemporains à la civilisation occidentale et à la colonisation. Suivant une logique essentialiste d'une utopique unité raciale noire, ce régime a besoin « de donner l'image d'une culture raciale cohérente et stable pour établir et légitimer le nationalisme politique noir et les notions de particularisme ethnique sur lesquelles il se fonde $»^{57}$. La culture

55. OBASANJO O., « Foreword », in Festac'77, op cit., p. 6.

56. FINGESIO, op. cit., 1977, p. 8.

57. GILROY P., L'Atlantique noir. Modernité et double conscience, Paris, Éditions Amsterdam, 2010 [1993], p. 145. 
noire serait, d'après Senghor, « un ensemble de valeurs originales provenant des Noirs, exprimé dans leurs différentes sociétés nationales ${ }^{58}$. De ce fait, le régime est obligé de montrer des manifestations artistiques anciennes qui se veulent authentiques, une tradition pure et non corrompue. Les gouvernants doivent inventer, avec l'aide d'artistes et d'intellectuels, des traditions nationales fantasmées : «le patrimoine culturel du peuple noir et africain est le point central de notre unité et de notre force. Ce patrimoine culturel englobe notre monde des arts, nos chants, nos danses, notre comportement vis-à-vis des autres, nos efforts communs et notre humanisme ${ }^{59}$, affirme Fingesi.

Le discours du régime sur la musique renforce l'idée d'une continuité du pouvoir et des pratiques précoloniales africaines dans le présent. Dans la publication officielle du FESTAC, l'ethnomusicologue Nwabena Nkeita affirme ainsi que « la musique traditionnelle - le patrimoine de l'Afrique contemporaine - est associé fortement à des institutions traditionnelles africaines de l'époque précoloniale ${ }^{60}$. Cette musique serait pour lui « une musique qui a survécu à l'impact des forces de formes occidentales d'acculturation, et, par conséquent, tout à fait distincte dans le langage et l'orientation de la musique populaire et de la musique savante contemporaine $»^{61}$. Ainsi, la musique traditionnelle des peuples du Nigeria et d'Afrique présentée au FESTAC serait un vestige authentique du passé précolonial et une preuve de la résistance de la culture noire. D’une façon générale, les catégories identitaires mobilisées par les régimes mexicain postrévolutionnaire et nigérian postcolonial au sein de leurs stratégies de légitimation visent le maintien de l'ordre social en soulignant la continuité ou l'héritage historique des régimes dans une prétendue unité nationale. Les références aux notions de «peuple», de « révolution » ou de « race » remplissent le présent d'un passé reconstruit et mythifié. Concernant l'Afrique postcoloniale, l'abus dans l'emploi de ces catégories est révélateur de comment « la nation, le peuple, l'unité sont érigés en principes politiques fondamentaux car ils soulignent en creux combien les sociétés africaines sont construites sur la division, la fragmentation, la faiblesse d'un sens collectif pourtant magnifié » ${ }^{62}$. Cette idée peut s'appliquer également à l'Amérique latine, où l'indigénisme est érigé en principe unificateur et rédempteur du passé colonial. Les régimes mexicain et nigérian nationalisent ainsi la violence symbolique exercée par la colonisation en réécrivant « leur » histoire coloniale.

58. SENGHOR L., « Black Culture », in Festac'77, op. cit., p. 12.

59. FINGESI O., Ibid., 1977, p. 9.

60. NKETIA K., « Music in African Culture », in Festac'77, op. cit., p. 20.

61. Ibid.

62. BOURMAUD D., La politique en Afrique, Paris, Montchrestien, 1997, p. 77. 


\section{Alliances internationales et réceptions nationales des stratégies de légitimation}

Les compositeurs, les hommes politiques ou les intellectuels peuvent entrer en conflit avec les stratégies de légitimation politiques ou les catégories identitaires mobilisées par les régimes, proposant d'autres catégories et innovant des stratégies en fonction des enjeux idéologiques et de leur position au sein du champ artistique et politique. Les conflits internes aux institutions du régime nous parlent des caractéristiques et du fonctionnement de ceux-ci ; les catégories esthétiques et les rituels mémoriels nous racontent les mythes et les passions politiques de la société en question. Ainsi, la conception primitiviste des arts mobilisée par le régime nigérian n'est pas partagée par tous les hommes politiques et ni par tous les artistes du pays, certains d'entre eux montrant leur ferme opposition. Le chef yoruba Obafemi Awolowo déclare ainsi à propos des préparatifs du FESTAC dès 1975 : « Je ne sais pas ce que le pays cherche à accomplir à travers un festival culturel. [II] n'est pas nécessaire pour la nation de faire revivre toutes les cultures primitives pour que nous les voyions. Nous voulons aller vers l'arrière tout simplement parce que nous ne savons pas comment aller de l'avant ${ }^{63}$. Dans une perspective critique du néocolonialisme affiché par le régime militaire, Awolowo affirme que l'Occident blanc est « intéressé à nous regarder démontrer notre primitivité », concluant avec ironie : « Nous devrions continuer à améliorer notre savoir-faire technologique et non pas à montrer combien nous sommes primitifs $»{ }^{64}$.

Cependant, l'enjeu international du FESTAC pour le régime est d'installer le Nigeria à la tête du continent africain et de la diaspora noire ainsi que d'accroître son influence au sein de la communauté internationale grâce à son identification comme le nouveau centre mondial de réflexion sur la culture noire. Ce projet répond en grande partie aux revendications des militants noirs états-uniens, qui voient dans l'Afrique la patrie commune de laquelle ils ont été arrachés par l'impérialisme occidental. Ainsi, Stokely Carmichael - idéologue du Black Power et dirigeant historique du Black Panthers Party - affirme dès 1967 que «c'est dans la mesure où les Noirs américains pourront se reconnaître "originaires" d'Afrique qu'ils seront capables d'avoir une action efficace sur la scène politique ${ }^{65}$. Néanmoins, la légitimité internationale du régime nigérian est ébranlée depuis la guerre civile déclenchée par le refus du gouvernement fédéral d'accepter l'indépendance et la libre détermination de la République du Biafra, région à

63. « So Far, So Wonderful », Daily Times, Lagos, December 7, 1975, p. 1, cité dans VEAL M., Fela. The Life and Times of an African Musical Icon, Philadelphia, Temple University Press, 2000, p. 274.

64. Ibid.

65. CARMICHAEL S., Le Black Power, Paris, Payot, 2009 [1967], p. 83. 
population majoritairement Ibo ${ }^{66}$. Les nombreuses critiques internationales contre l'action du régime nigérian sont renforcées par la médiatisation de la famine provoquée par le blocus mené par l'armée fédérale dans des zones dominées par l'armée sécessionniste.

Ainsi, l'objectif avoué du régime lors du FESTAC est d'inaugurer une nouvelle ère de la culture et de la civilisation noire dans le monde, facilitant le retour aux origines des artistes et des écrivains de la diaspora. En effet, le caractère panafricaniste du FESTAC viserait « à inclure tous les éléments humains hétérogènes identifiables dans le monde noir et africain, ainsi que chez tous les peuples du monde avec un héritage africain ${ }^{67}$. Pour le régime d'Obasanjo, « rien n'est plus approprié en ce moment dans l'Histoire noire et africaine que la redécouverte des liens culturels et spirituels qui unissent tous les peuples noirs et africains dans le monde entier ${ }^{68}$. Obasanjo lui-même assure que « c'est la pleine réalisation de ce fait qui a motivé le Gouvernement Fédéral Militaire à prendre la responsabilité de l'organisation et la tenue du Second World Black and African Festival of Arts and Culture ${ }^{69}$. Le régime nigérian reçoit des délégations de plus de soixante pays du continent africain ou reliés à l'Afrique par des liens historiques en rapport avec l'arrivée d'esclaves sur leur territoire. Le FESTAC est ainsi l'occasion pour le régime de se montrer comme le pays africain capable d'affronter « l'Occident impérialiste » et de fédérer les revendications noires du monde entier. Par le biais d'un renversement symbolique, Obasanjo prend le soin de déclarer que «l'objectif du Festival n'est pas de sous-estimer ou de rabaisser les valeurs culturelles des autres races. Au contraire, nous cherchons à déployer nos valeurs dans un monde hautement compétitif où le patrimoine culturel noir et africain peut coexister avec les valeurs culturelles des peuples d'autres pays sans conflit majeur. En d'autres termes, nous recherchons l'harmonie culturelle fondée sur la dignité humaine et le respect mutuel $»^{70}$.

66. Membre de l'ONU depuis le 7 octobre 1960, le Nigeria adopte pourtant lors de l'Assemblée générale des Nations Unis du 14 décembre de la même année la Déclaration sur l'octroi de l'indépendance aux pays et aux peuples coloniaux, dont le second article affirme : « Tous les peuples ont le droit de libre détermination ; en vertu de ce droit, ils déterminent librement leur statut politique et poursuivent librement leur développement économique, social et culturel ». De même, le septième article déclare : « Tous les États doivent observer fidèlement et strictement les dispositions [...] de la présente Déclaration sur la base de l'égalité, de la non-ingérence dans les affaires intérieures des États et du respect des droits souverains et de l'intégrité territoriale de tous les peuples », $c f$. « Déclaration sur l'octroi de l'indépendance aux pays et aux peuples coloniaux », Résolution 1514 (XV) adopté le 14 décembre 1960 dans la $947^{\mathrm{e}}$ séance plénière de l'Assemblée générale des Nations Unies.

67. OBASANJO O., op. cit., 1977, p. 6.

68. Ibid.

69. Ibid.

70. Ibid., p. 7. 
Cependant, le FESTAC est vécu par de nombreux Nigérians comme le gaspillage inutile et extravagant d'un régime corrompu et sourd aux besoins des citoyens. Les efforts du régime pour construire symboliquement une nation fédérale, modèle de respect et de valorisation des différents peuples qui la forment, ne sont pas accompagnés par des réformes économiques et sociales qui permettent d'élever le niveau de vie des plus démunis. La dépendance nationale aux recettes et à la production pétrolières néglige d'autres secteurs nécessaires à l'équilibre de l'économie du pays : l'agriculture et l'industrie déclinent dramatiquement durant la décennie de 1970, l'inflation des produits alimentaires est de $273 \%$ entre 1973 et $1981^{71}$. Ainsi, l'efficacité réelle des stratégies de légitimation symbolique du régime se voit limitée par la pauvreté et par l'absence des perspectives politiques de la population.

Contrairement au régime nigérian, le Général Lázaro Cárdenas entreprend de nombreuses réformes politiques et sociales à son arrivée au pouvoir au Mexique : le rétablissement des relations diplomatiques avec l'URSS - rompues par ses prédécesseurs en 1930 -, la légalisation du Parti Communiste Mexicain (PCM), le respect des droits syndicaux et des revendications ouvrières, la formation d'un Front Populaire Mexicain ${ }^{72}$ (FPM) et la nationalisation des industries minières et pétrolières. Ces réformes répondent aux attentes d'une grande partie des acteurs sociaux - des intellectuels, des artistes et des grandes centrales syndicales paysannes et ouvrières -, qui réclament l'accomplissement des revendications populaires de la Révolution.

Ces réformes sont accompagnées d'une politique symbolique qui mobilise des catégories marxistes, identifiant la Révolution mexicaine à la Révolution d'octobre. Le régime continue son utilisation de la musique dans des actes mémoriels et politiques, intégrant désormais la lutte de classes et anti-impérialiste dans sa rhétorique. Le Département des Beaux-Arts de la SEP fonde en 1935 un nouvel orchestre subventionné entièrement par l'État, l'Orchestre Symphonique National (OSN), en concurrence avec à l'OSM de Carlos Chávez qui est financé majoritairement par une fondation privée. Silvestre Revueltas assure la direction de l'OSN et participe à une nouvelle série d'actes politiques du régime afin de réactiver l'héritage de la Révolution par l'idéologie marxiste. Revueltas dirige ainsi L'Internationale à la tête de l'OSN au rassemblement organisé par la Société des Amis de l'URSS pour commémorer le $18^{\mathrm{e}}$ anniversaire de la Révolution Russe ${ }^{73}$.

71. FALOLA T., HEARTON M., op. cit., 2008, p. 183.

72. Le FPM est une formation politique et syndicale fondée en 1936 avec le soutien de l'État afin de soutenir les réformes sociales du gouvernement de Lázaro Cárdenas et de transformer le régime postrévolutionnaire dans une optique marxiste.

73. El Nacional, México, 6 novembre 1935. 
Quelques semaines plus tard, Revueltas participe à la grande manifestation organisée par le gouvernement Cárdenas à Mexico, au Palacio de Bellas Artes le 20 novembre 1935, pour commémorer le $25^{\mathrm{e}}$ anniversaire de la Révolution mexicaine ${ }^{74}$. Revueltas dirige à la tête de l'OSN et du Chœur du Conservatoire un programme constitué notamment par Caminos de Revueltas lui-même et par la Symphonie $n^{\circ} 3$ «Premier Mai » de Dimitri Chostakovitch. La fonction symbolique de l'œuvre de Revueltas relie désormais la famille révolutionnaire, l'identité mexicaine métisse et la lutte de classes dans une dimension internationale.

En revanche, les discours essentialistes et primitivistes sur la musique mobilisés par le régime nigérian lors du FESTAC - afin d'établir une hypothétique continuité entre les traditions précoloniales, le pouvoir du régime et celui des autorités traditionnelles des différentes régions du Nigeria - ne sont pas soutenus par un projet de transformation sociale. L'essentialisme et la corruption du régime sont dénoncés comme une sorte de néocolonialisme et de ce fait critiqués par certains artistes, notamment Fela Kuti. Pour lui, le FESTAC n'est qu'une campagne démagogique et corrompue du régime militaire qui vise à maintenir l'ordre social et à protéger les intérêts du régime. Il prend soin d'annoncer sa démission du comité organisateur du FESTAC, auquel il ne veut pas être assimilé. Lors d'un entretien, Fela exprime les raisons de cette décision, en déclarant : « Le FESTAC ! Une vraie connerie, man ! Une pure escroquerie ! Ils ont essayé de me plonger dedans. Ils ont commencé par être "gentils" avec moi... Tu vois ? J'ai été invité à assister à la réunion du Comité national nigérian de préparation du festival. [...] [Le major général I. B. M. Haruna] a commencé avec toute une putain de démagogie, disant qu'il était “ouvert" aux idées neuves, et tout ça. Je lui ai donc soumis un programme d'action en neuf points pour que le festival devienne quelque chose d'authentique : une affaire du peuple ! Le premier point de mon programme était la participation du peuple. Puis - je dénonçais les magouilles en cours ; la manière triviale dont les cultures des peuples du Nigeria étaient traitées, et tout ça ? Le major général Haruna a rejeté en bloc toutes mes propositions. Du coup j'ai démissionné du comité préparatoire ${ }^{75}$.

Fela mobilise la notion de «peuple » comme élément identificatoire de sa création musicale et de sa vision politique. En effet, fondée sur l'idéologie à la fois panafricaniste de Kwame Nkrumah et émancipatrice du Black Power, sa musique s'approprie la soul et le funk des États-Unis ainsi que le jazz pour fonder une modernité musicale noire - l'afro-beat - qui ne puiserait pas son authenticité dans un imaginaire précolonial. Fela fait partie

74. El Nacional, México, 15 novembre 1935.

75. MOORE C., Fela, Fela, cette putain de vie, Paris, Karthala, 1982, p. 141. 
des compositeurs qui enracinent leur musique dans une modernité occidentale - il étudie au Trinity College de Londres de 1958 à 1962 puis séjourne aux États-Unis entre 1969 et 1970 - en cherchant leur appartenance et leur spécificité à l'intérieur des mouvements d'émancipation noirs africains et occidentaux, notamment les Black Panthers. Il se place délibérément dans une critique frontale du pouvoir, dans sa musique et dans son discours, recherchant une « africanité » propre au destin postcolonial de l'Afrique. De fait, comme acte symbolique d'émancipation postcoloniale, il change en 1975 son nom d'esclave "Ransome », suivant l'exemple de Malcom $\mathrm{X}^{76}$, pour le nom yoruba « Anikulapo » qui signifie « avoir la mort dans son carquois $\gg{ }^{77}$. La musique serait pour Fela une arme capable d'éveiller la conscience du peuple et d'enclencher un mouvement d'émancipation symbolique et politique.

Fela ne participe à aucune manifestation organisée par le FESTAC et organise dans sa propre salle de concert, le Shrine (le Temple), des manifestations quotidiennes afin de dénoncer la corruption et l'idéologie néocolonialiste du régime. Parmi les invités les plus prestigieux qui passent leurs nuits au Shrine se trouvent Stevie Wonder, Gilberto Gil, Archie Shepp, les membres du Sun Ra Arkestra et de l'Art Ensemble of Chicago. Fela raconte : « je suis resté au Shrine, pour faire mon contre-FESTAC. Car, tu vois, tous les grands musiciens et artistes que le FESTAC a fait venir voulaient me rencontrer. Pendant un mois, nuit après nuit, le Shrine était bourré à craquer de Noirs venus de partout. Ils voulaient tous savoir ce que se passait réellement au Nigeria. Pourquoi je boycottais le Festival ? etc. Et je leur ai tout dit. J'ai utilisé le Shrine comme une tribune pour dénoncer toute la merde, toute la corruption de ce gouvernement qui les avait invités. J'ai tout déballé ${ }^{78}$.

En plus de dénoncer la corruption du régime, Fela Kuti ridiculise l'armée, avec sa chanson Zombie, en comparant les soldats à des zombies obéissant aveuglément aux ordres des supérieurs. Le prix payé par Fela Kuti est élevé car, une fois le FESTAC terminé, le régime ordonne à l'armée régulière de prendre d'assaut le 18 février la maison du chanteur - la République de Kalakuta. L'importance que le régime octroie à ces critiques et le recours à la violence afin de les faire taire montre la faiblesse des institutions régulatrices du régime - justice, instances culturelles officielles, industrie culturelle - ainsi que la fragilité de sa légitimé internationale. Fela finit par s'exiler au Ghana durant l'été 1977 pour retourner à Lagos en 1978 et

76. Le militant noir états-unien Malcolm X (1925-1965) abandonne son patronyme « Little », le patronyme du propriétaire de ses pères, pour X, le nom de personne. Ce geste lui permet de se libérer symboliquement de son héritage d'esclave et de renouer avec son passé.

77. MOORE C., op. cit., 1982, p. 137.

78. Ibid., p. 142. 
fonder son propre parti politique, le Movement of the People ${ }^{79}$ (M.O.P.), afin d'essayer de briguer le pouvoir - sans succès - lors des élections de 1983.

Contrairement au cas nigérian, le régime de Cárdenas compte avec le soutien des artistes et des intellectuels proches du Parti Communiste Mexicain et des membres de la Liga de Escritores y Artistas Revolucionarios ${ }^{80}$ (Ligue des Écrivains et Artistes Révolutionnaires, LEAR), dont Silvestre Revueltas préside le Comité Exécutif entre mai 1936 et février 1937. Le parti officiel, le Partido Nacional Revolucionario ${ }^{81}$ (Parti National Révolutionnaire, PNR), organise conjointement avec la LEAR, le 5 juillet 1936 au Palacio de Bellas Artes, un hommage à la mort de l'écrivain russe Maximo Gorki ${ }^{82}$. Parmi les orateurs de cet hommage officiel se trouve notamment le dramaturge espagnol Cipriano de Rivas Cherif ${ }^{83}$. Revueltas y participe dirigeant à nouveau sa musique devant l'OSN. Quelques jours plus tard, le 18 juillet 1936, a lieu en Espagne le soulèvement militaire qui marque le début de la guerre civile (1936-1939). L'adhésion des intellectuels antifascistes de la LEAR aux actions du régime est totale quand celui-ci décide de soutenir la II ${ }^{\text {ème }}$ République espagnole. La direction du PNR émet un communiqué de soutien au gouvernement républicain le 19 juillet, avec une empathie révélatrice des enjeux nationaux de son identification internationale : «Le Parti National Révolutionnaire, défenseur des régimes qui s’identifient aux classes travailleuses, souhaite la consolidation définitive du gouvernement espagnol, au moment où il est menacé par la trahison des militaires. La victoire démocratique d'un gouvernement qui menace les anciens privilèges concrétiserait les réformes sociales qui unissent nos nations, intimement

79. Fela revendique l'héritage de Nkrumah dans l'idéologie du M.O.P. et, paradoxalement, il intègre une grande partie des recommandations prises lors du colloque international du FESTAC. Sur ce sujet voir VEAL M., op. cit., 2000, p. 169.

80. La LEAR est une organisation mexicaine d'intellectuels et d'artistes antifascistes. Active de 1934 à 1938, elle se donne pour mission l'impulsion de la lutte des classes par la création artistique afin de faire advenir une société plus juste sous l'idéologie marxiste. Lors de sa fondation en 1934, elle se définit comme une « organisation de Frente Único au service de la classe travailleuse contre l'actuel système capitaliste et ses funestes conséquences de crise et dégénérescence avec ses séquelles de fascisme, d'impérialisme et de guerre ». À partir de 1936, elle agit pour la lutte mondiale antifasciste et travaille activement avec le régime postrévolutionnaire mexicain. Sur l'histoire de la LEAR voir VELASCO PUFLEAU L., « La Liga de Escritores y Artistas Revolucionarios (LEAR) : 1'antifascisme communiste au Mexique », Revue Aden, Bruxelles, n 10, 2011, p. 184-204.

81. La création du Parti National Révolutionnaire en mars 1929 constitue l'acte fondateur de l'institutionnalisation progressive du régime autoritaire postrévolutionnaire et le début du processus de démilitarisation du système politique mexicain. Sur l'histoire politique de l'État mexicain voir MEDINA PEÑA L., Hacia el nuevo Estado. México, 1920-2000, México, FCE, 2010.

82. Gorki décède à Moscou le 18 juin 1936.

83. Le dramaturge et metteur en scène Cipriano de Rivas Cherif (1891-1967) collabore avec les compagnies de théâtre les plus importantes de l'Espagne des années 1920 et 1930. Ami de Federico García Lorca, il crée La Zapatera prodigiosa (1930) et Yerma (1934) avec la compagnie de Margarita Xirgu, alors concessionnaire du Teatro Español de Madrid entre 1930 et 1935 . Frère de Dolores Rivas Cherif, la femme du Président de la République espagnole Manuel Azaña, Cipriano de Rivas Cherif est consul général à Genève durant la Guerre civile espagnole. 
liées par le destin historique et par la lutte contre toute forme d'exploitation inhumaine $[\mathrm{sic}] »^{84}$.

Ainsi, le régime mexicain réussit à fédérer les artistes, les intellectuels et les organisations du Front Populaire Mexicain dans une union nationale qui impliquerait une lutte internationale démocratique et antifasciste. Néanmoins, dès la fin 1937 le régime ne permet pas que le projet de construction nationale soit mobilisé par les syndicats, réunis sous la bannière du FPM, par le biais d'une rhétorique marxiste capable de remplacer la mobilisation identitaire au nom du métissage et de l'indigénisme. Lázaro Cárdenas consolide le régime autoritaire postrévolutionnaire transformant en mars 1938 le PNR en un parti de type corporatiste, le Partido de la Revolución Mexicana $^{85}$ (Parti de la Révolution Mexicaine, PRM). Le nouveau parti hégémonique incorpore le FPM, l'armée et divers mouvements politiques dans ses quatre « secteurs »: le secteur ouvrier, le secteur paysan, le secteur populaire et le secteur militaire. La domination totale du paysage politique par le Parti de la Révolution Mexicaine rend la compétition politique impossible durant plusieurs décennies. De même, la fondation du PRM marque la fin du projet marxiste de construction nationale soutenu par la LEAR et le PCM tout en institutionnalisant une éternelle Révolution sociale.

\section{Conclusion}

Les stratégies de légitimation symbolique déployées au sein des dispositifs analysés dans les deux études de cas visent à établir des catégories discursives dans le but de maintenir - ou contester - un certain ordre social grâce à l'imaginaire et aux rituels qui instaurent ou sacralisent des temporalités et des mémoires ${ }^{86}$. L'instauration d'une nouvelle mémoire historique dans l'espace public est en grande partie réalisée par des rituels mémoriels où la musique occupe une place privilégiée par sa capacité à enclencher des processus d'identification. La mobilisation d'outils symboliques peut exacerber ou effacer les ruptures et les conflits entre les différents régimes au profit du pouvoir en place. Comme nous l'avons vu, l'utilisation de ressources symboliques peut se révéler d'une grande efficacité dans la légitimation du

84. El Nacional, México, 19 juillet 1936, reproduit dans OJEDA REVAH, M., México y la Guerra civil española, Madrid, Turner, 2004, p. 103-104.

85. Le PRM deviendra à son tour le Parti Révolutionnaire Institutionnel (PRI) en 1946 et restera au pouvoir jusqu'en 2000. Il y est revenu lors des élections présidentielles de 2012.

86. Martine Segalen souligne avec justesse que « les rites et rituels donnent à nos sociétés ordre et hiérarchie par le biais d'actions qui créent du sens, qui suscitent des émotions collectives, qui aident à interpréter le monde. Leur potentiel créatif permet d'installer des temporalités et des mémoires », SEGALEN M., Rites et rituels contemporains, Paris, Armand Colin, 2009, p. 11. 
pouvoir par la réactivation du passé et par son rattachement à une tradition imaginée, à condition d'être accompagnée par des réformes économiques et sociales et politiques. En effet, la légitimité durable tiendrait à l'alliance entre plusieurs types de stratégies qui vont des pratiques économiques ${ }^{87}$ aux opportunités politiques en passant par le renforcement du sentiment d'appartenance, symbolique et physique, au groupe social. Ainsi, l'efficacité symbolique du FESTAC en tant que dispositif de légitimation interne du régime est certainement limitée. La faiblesse du système d'éducation nationale et l'absence d'institutions régulatrices de la création et de la diffusion musicale ne permettent pas au régime nigérian de renforcer les catégories identitaires mobilisées lors du FESTAC par le biais des programmes scolaires et d'œuvres musicales fédératrices. La production et l'institutionnalisation d'une nouvelle Histoire nationale aurait nécessité, comme dans le cas du Mexique, un travail conjoint entre le régime, les institutions artistiques et le système éducatif afin de l'instaurer et de la transmettre à toute une génération, de l'enseignement primaire à l'université.

L'une des différences les plus importantes entre les politiques culturelles qui permettent la mise en place du dispositif de légitimation symbolique des deux régimes est le soutien étatique au développement d'un art et d'une musique qui seraient représentatifs de la communauté nationale. Le régime mexicain postrévolutionnaire utilise symboliquement la musique symphonique soutenue par les institutions de l'État lors des rituels politiques. En revanche, l'absence d'un processus plus large de construction nationale au Nigeria empêche que les symboles et les catégories mobilisés lors du FESTAC s'installent durablement dans les discours et les représentations. De même, le discours officiel mobilisé par le régime nigérian à propos de la musique impose une vision monolithique et immuable de la culture noire qui, cherchant son authenticité exclusivement dans l'héritage précolonial, ne favorise pas la recherche de la spécificité des États-nations africains indépendants depuis seulement quelques années. La polysémie de la musique permet aux régimes d'investir celle-ci de traits nationaux en fonction des catégories mobilisées dans l'espace publique. Cependant, d'une façon générale, ces traits sont construits socialement afin de souligner la spécificité de la Nation et peuvent présenter des contradictions inhérentes aux formes, au langage et au matériau musicaux. Le processus de construction nationale cherche ainsi à s'approprier l'héritage colonial par l'intériorisation des symboles et la différenciation des pratiques. Comme le remarque Adorno, « depuis le milieu du dix-neuvième siècle, les musiques sont devenues des idéologies politiques du fait qu'elles ont mis en avant des traits nationaux, qu'elles se sont données pour représentantes des nations et qu'elles ont

87. Comme le montre le travail de Béatrice Hibou sur l'analyse de la domination à partir d'une approche d'économie politique (HIBOU B., Anatomie politique de la domination, Paris, La Découverte, 2011). 
confirmé en tout lieu le principe national. Mais, plus qu'aucun autre support artistique, la musique donne forme en elle-même aux antinomies du principe national $\gg^{88}$.

Dans le cas du Mexique, le discours rétrospectif de l'historiographie officielle des années 1930 développe l'idée que la Nation mexicaine accède à la modernité musicale grâce au travail des compositeurs, qui l'ont dotée d'une musique nationale à caractère universel. Le Mexique se serait trouvé lui-même pour la première fois, par un processus de différenciation identitaire, grâce à un patrimoine symbolique commun capable de fédérer la Nation. Le régime nigérian conçoit quant à lui son destin national au sein d'un panafricanisme idéalisé et relativement homogène. L'accession du Nigeria au premier plan de la scène internationale constituerait la reconnaissance des apports de l'Afrique à la civilisation occidentale, et vice-versa. L'espoir soulevé par le FESTAC dans l'imaginaire noir des deux côtés de l'Atlantique est incontestable. Aux États-Unis, la revue The Black Perspective in Music rapporte le témoignage d'un participant qui n'hésite pas à affirmer : «Il y a désormais un nouvel espoir. L'important est que les Noirs ont été rassemblés ; personne ne partira d'ici sans réfléchir sérieusement à ces questions importantes. Ce festival culturel nous a rendus conscients et nous a donné l'opportunité de concevoir un programme fonctionnel pour finir le siècle ${ }^{89}$.

88. ADORNO T., Introduction à la sociologie de la musique, Genève, Contrechamps, 1994 [1962], p. 160.

89. « FESTAC '77 », The Black Perspective in Music, vol. 5, n’ 1, 1977, p. 104-105. 